\title{
What is the source of the magnetic helicity shed by CMEs? The long-term helicity budget of AR 7978
}

\author{
P. Démoulin ${ }^{1}$, C. H. Mandrini2 ${ }^{2}$ L. van Driel-Gesztelyi ${ }^{1,3,4,5}$, B. J. Thompson ${ }^{6}$, \\ S. Plunkett ${ }^{7}$, Zs. Kövári ${ }^{4}$, G. Aulanier ${ }^{1}$, and A. Young ${ }^{6}$ \\ 1 Observatoire de Paris, section Meudon, DASOP, URA 2080 (CNRS), 92195 Meudon Principal Cedex, France \\ 2 Instituto de Astronomía y Física del Espacio, IAFE, CC. 67 Suc. 28, 1428 Buenos Aires, Argentina* \\ e-mail: mandrini@iafe.uba.ar \\ 3 Mullard Space Science Laboratory, Univ. College London, UK \\ 4 Konkoly Observatory, Hungary \\ 5 Centre for Plasma Astrophysics, K.U. Leuven, Celestijnenlaan 200B, 3001 Heverlee, Belgium \\ 6 Goddard Space Flight Center - NASA, USA \\ 7 USRA, Naval Research Laboratory, Washington, DC 20375, USA
}

Received 3 October 2001 / Accepted 15 November 2001

\begin{abstract}
An isolated active region (AR) was observed on the Sun during seven rotations, starting from its birth in July 1996 to its full dispersion in December 1996. We analyse the long-term budget of the AR relative magnetic helicity. Firstly, we calculate the helicity injected by differential rotation at the photospheric level using MDI/SoHO magnetograms. Secondly, we compute the coronal magnetic field and its helicity selecting the model which best fits the soft X-ray loops observed with SXT/Yohkoh. Finally, we identify all the coronal mass ejections (CMEs) that originated from the AR during its lifetime using LASCO and EIT/SoHO. Assuming a one to one correspondence between CMEs and magnetic clouds, we estimate the magnetic helicity which could be shed via CMEs. We find that differential rotation can neither provide the required magnetic helicity to the coronal field (at least a factor 2.5 to 4 larger), nor to the field ejected to the interplanetary space (a factor 4 to 20 larger), even in the case of this AR for which the total helicity injected by differential rotation is close to the maximum possible value. However, the total helicity ejected is equivalent to that of a twisted flux tube having the same magnetic flux as the studied AR and a number of turns in the interval $[0.5,2.0]$. We suggest that the main source of helicity is the inherent twist of the magnetic flux tube forming the active region. This magnetic helicity is transferred to the corona either by the continuous emergence of the flux tube for several solar rotations (i.e. on a time scale much longer than the classical emergence phase), or by torsional Alfvén waves.
\end{abstract}

Key words. Sun: corona - Sun: coronal mass ejections (CMEs) - Sun: magnetic fields - solar-terrestrial relations

\section{Introduction}

Coronal mass ejections (CMEs) are episodic expulsions of mass and magnetic field from the solar corona into the interplanetary medium, producing significant perturbations in the solar wind and the geomagnetic environment. These huge ejected plasmoids may have masses of the order of a few $10^{15} \mathrm{~g}$ and may liberate energies between $10^{30}$ $10^{32}$ ergs. CMEs were first identified in data obtained with space-borne coronagraphs in the 70's (Mac Queen 1980; Sheeley 1980; Mac Queen et al. 1991), and since then they

Send offprint requests to: P. Démoulin,

e-mail: pascal.demoulin@obspm.fr

* Member of the Carrera del Investigador Científico, CONICET, Argentina. have captured the attention of both the solar and the geomagnetic communities. Several excellent review papers have been written in the last years (Crooker et al. 1997; Hundhausen 1999; Pick et al. 1999; Forbes 2000; Klimchuk 2000; Webb 2000; Hudson \& Cliver 2001). One of the most recent and challenging works on theoretical and observational aspects of CMEs is the "Q\&A" review by Cliver \& Hudson (2001).

CMEs are thought to be the last physical mechanism in the chain of processes which transfer magnetic flux and helicity from the base of the convective zone (from the dynamo region) into the interplanetary medium. Both magnetic flux and helicity are injected from the convective zone into the corona in a systematic, though bursty, way. Magnetic flux reverses its sign with the solar cycle while 
magnetic helicity does not (in a given solar hemisphere). Because magnetic helicity dissipation is a very slow process (Berger 1984), the Sun can avoid endless accumulation of helicity in the solar corona only by ejecting the accumulated helicity via the launch of CMEs (Low 1997).

Theoretical MHD models of coronal arcades have shown that magnetic shear exceeding a critical threshold can lead to their destabilization (Mikić et al. 1994; Mikić \& Linker 1997), indicating that they can be considered candidates for the initiation of CMEs. Reconnection occurring in a current sheet within the arcade could then produce not only newly closed arcades, but also a twisted flux rope which is expelled from the corona. Several other CME models invoke the presence of twisted flux ropes in the corona before the ejection. These models show that a slow photospheric evolution (Forbes \& Priest 1995; Lin et al. 1998), or a fast injection of new magnetic flux (Chen 1996), can lead to the ejection of the magnetic flux rope. All these models, thus, suggest that the observed interplanetary magnetic clouds are due to the ejection of magnetic flux ropes from the corona (seen as CMEs).

Interplanetary magnetic clouds are formed by twisted magnetic flux tubes, as revealed by "in situ" measurements of their magnetic fields (Burlaga 1981; Burlaga et al. 1988; Lepping et al. 1990; Hidalgo et al. 2000; Shimazu \& Marubashi 2000). These clouds have often been associated with disappearing filaments on the Sun (Marubashi 1986; Burlaga et al. 1998; Srivastava et al. 1998). The magnetic flux rope, together with the disappearing filament, is thought to be ejected from the Sun in the form of a CME that traverses the interplanetary medium carrying magnetic flux and helicity with it. The computation of the helicity in magnetic clouds can give us clues concerning the amount of helicity shed by CMEs, a quantity which is useful to compare to the helicity injected and stored in the corona.

The life of active regions (ARs) reflects the evolution of the magnetic field that emerges through the photosphere, interacts with the pre-existing magnetic environment, becomes dispersed by large-scale flows and supergranular or granular convection and, furthermore, is sheared by the differential rotation. Once an AR appears at the photospheric level, it reaches its maximum development in a few days. Compared to this short emergence period, its decay time can be much longer, of the order of months (see the review by van Driel-Gesztelyi 1998). During this decay phase flaring, which might have been prolific and violent during the AR growth, diminishes considerably, while CMEs may well occur accompanied by the eruption of long filaments. The total number of CMEs produced by one single active region during its entire lifetime, from the cradle to the grave, can give us information about the total amount of magnetic helicity the AR contains. However, during solar maximum, both because there are several active regions on the Sun and because a significant part of the CMEs are launched outside ARs, it is difficult to make a full CME count for a source region, especially, when it is behind the limb of the Sun. Periods of low solar activity provide a better opportunity for such study.

Solar activity during the second half of 1996 was governed by a single AR, which was located on the southern hemisphere. This region was identified as NOAA AR 7978 when it first appeared on July 6, 1996. From this month and for the next five solar rotations (until November 1996), only a few small, short-lived ARs were observed on the solar disk besides AR 7978. Thus, solar activity at that period came mainly from AR 7978, and a direct comparison between the evolution of the AR magnetic field and its flare/CME production can be made. This long-term (six months) study gives us the unique opportunity to investigate the origin of the helicity in the field, as generated by differential rotation or/and brought up from the convective zone by twisted flux emergence. Furthermore, the simple bipolar configuration of AR 7978, which is maintained throughout its life, makes the results clearer, since the studied configuration is relatively close to theoretical configurations: in the convective zone (emergence of a single flux tube), in the corona (no interaction with neighboring active regions) and for the injection of helicity by differential rotation (a bipolar field is the simplest configuration, still twist and writhe magnetic helicity injected by differential rotation nearly cancel as shown by Démoulin et al. 2001).

This paper is organized as follows: in Sect. 2 we describe the data used in our long-term analysis; then we present a global description of the evolution of AR 7978 at different atmospheric levels, together with a study of the evolution of the magnetic shear based on models of the coronal magnetic field (preliminary results can be found in van Driel-Gesztelyi et al. 1999; Mandrini et al. 2000). Magnetic helicity is defined in Sect. 3. In Sect. 4, we briefly describe the injection of magnetic helicity in the corona by differential rotation. After that, we present the computation of magnetic helicity in the coronal field (Sect. 5) and in interplanetary magnetic clouds (Sect. 6). We apply the results to AR 7978 and Sect. 7 describes its magnetic helicity budget. Finally, in Sect. 8, we discuss our results. We find that differential rotation is neither sufficient to generate the helicity found in the coronal field, nor the helicity ejected from the Sun.

\section{The long-term evolution of AR 7978}

\subsection{The data}

The data set used in the present paper consists of images obtained with instruments on board the SoHO and Yohkoh spacecrafts, among them the Michelson Doppler Imager (SoHO/MDI, Scherrer et al. 1995) the Extreme Ultraviolet Imaging Telescope (SoHO/EIT, Delaboudinière et al. 1995), the Large Angle Spectroscopic Coronagraph (SoHO/LASCO, Brueckner et al. 1995) and the Soft X-ray Telescope (Yohkoh/SXT, Tsuneta et al. 1991). In particular, we have analyzed full-disc 5-min averaged magnetograms from SoHO/MDI, images taken with 


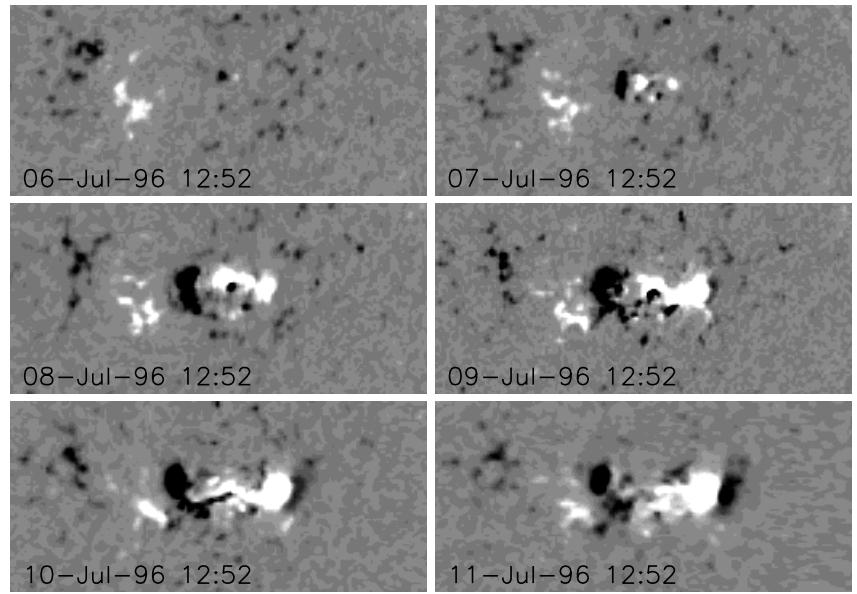

Fig. 1. Evolution of the longitudinal magnetic field during the emergence of AR 7978 (white: positive polarity, black: negative polarity). The spatial coordinates have been corrected from projection effects. The negative polarity (black) which grow in front of the main positive polarity is the classical evolution of the longitudinal magnetic field as an AR rotates towards the west limb.
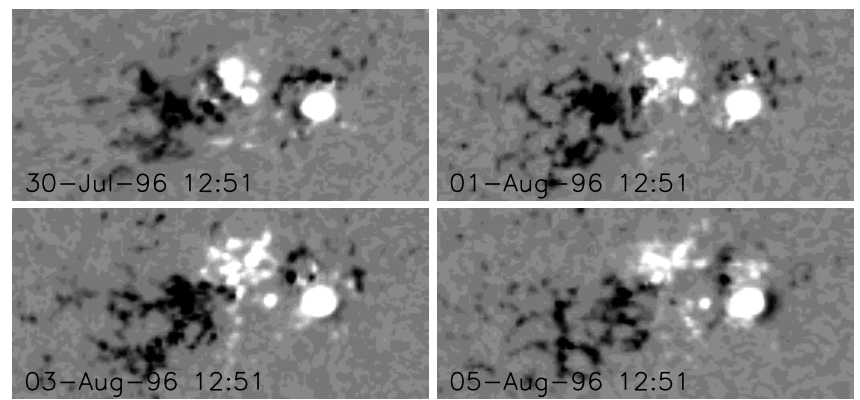

Fig. 2. Evolution of the longitudinal magnetic field during the second rotation of AR 7978 over the solar disk (same convention as in Fig. 1). The remnant field of the second emergence is present as a tilted bipole (the first bipole being oriented nearly east-west).

all the four SoHO/EIT filters (304 $\AA, 195 \AA, 171 \AA$ and $384 \AA$ ), SoHO/LASCO C2 and C3 white-light coronagraph observations, and full-disc and partial frame soft X-ray images from Yohkoh/SXT taken with the Al $0.1 \mu \mathrm{m}$ and the Dagwood AlMg filters.

\subsection{Evolution of the photospheric magnetic field}

Strong flux emergence started at S10 E31 on July 4, 1996. The new flux appeared in a dominant negative polarity environment (West of an old and dispersed bipolar region) and in front of a small decaying AR, as shown in Fig. 1. The new AR was named as NOAA AR 7978 and the fast growth of the magnetic flux and area lasted until at least July 10. As the AR grew, the old following positive flux (about $1.8 \times 10^{21} \mathrm{Mx}$ ) was completely swept out, and was eventually cancelled by the new negative flux (Fig. 1). We will call this period of time the beginning of the first rotation (which lasts till the AR rotates back to the central meridian), and we will identify the region as AR 7978 along all the following rotations; though in fact it was denoted as NOAA 7981 and NOAA 7986 in the second and the third rotations, respectively (Figs. 2 and 3). After the spots disappeared (from the fourth rotation on) the region did not have a NOAA number any longer, but since its magnetic field was still clearly present (Fig. 3), we could follow its evolution for three more rotations, and refer to it as AR 7978.

There was a second episode of flux emergence in the AR, which started while it was on the invisible side of the Sun and continued for a few days after the AR rotated onto the disk (Fig. 2). The new flux emergence increased the magnetic flux measured during the first rotation (total unsigned flux of $1.5 \times 10^{22} \mathrm{Mx}$ ) by about $50 \%$ $\left(2.3 \times 10^{22} \mathrm{Mx}\right)$, see Fig. 4. The peak flux was reached during the third rotation $\left(2.4 \times 10^{22} \mathrm{Mx}\right)$, this was due to an increase of the negative flux creating a roughly $10 \%$ flux imbalance in the AR. The imbalance was plainly due to the fact that the AR emerged in a dominantly negative polarity magnetic environment and, as its flux was getting diffused and its magnetic area grew, more and more of this pre-existing negative flux was included in the measurements (van Driel-Gesztelyi et al. 1999). Later the total flux decreased slowly, and even increased during the last two rotations due to an increasing level of small-scale new flux emergence and the ever-increasing area.

After the first two rotations the global deformation of the flux concentrations were mainly due to the effect of diffusion and differential rotation. Figure 3, right column, illustrates the long-term evolution of the magnetic field of AR 7978 at successive central meridian passages (CMPs). In the left column of the same figure, we show magnetic maps rotated to the time of the following CMP map, applying differential rotation. Comparing maps in the left and right columns, we observe the same tilt of the magnetic inversion line implying that the global deformation of the AR, as seen by the longitudinal magnetograms of MDI, was just the result of differential rotation.

\subsection{Evolution of the coronal loops}

The evolution of the soft X-ray coronal structures, out of the time of flare activity, depicts the typical evolution of an AR from emergence until death (van Driel-Gesztelyi et al. 1998). The soft X-ray intensity in Yohkoh/SXT images is high during the first rotation and then it slowly decreases, as the magnetic flux disperses. Figure 5 illustrates this evolution through images obtained at CMP from the second to the fifth rotation. The orientation of the SXT loops, compared to the direction of the inversion line (see Figs. 3 and 5), indicates the apparent increase of magnetic shear at coronal heights from the second to at least the fourth rotation (see Sect. 2.5). 

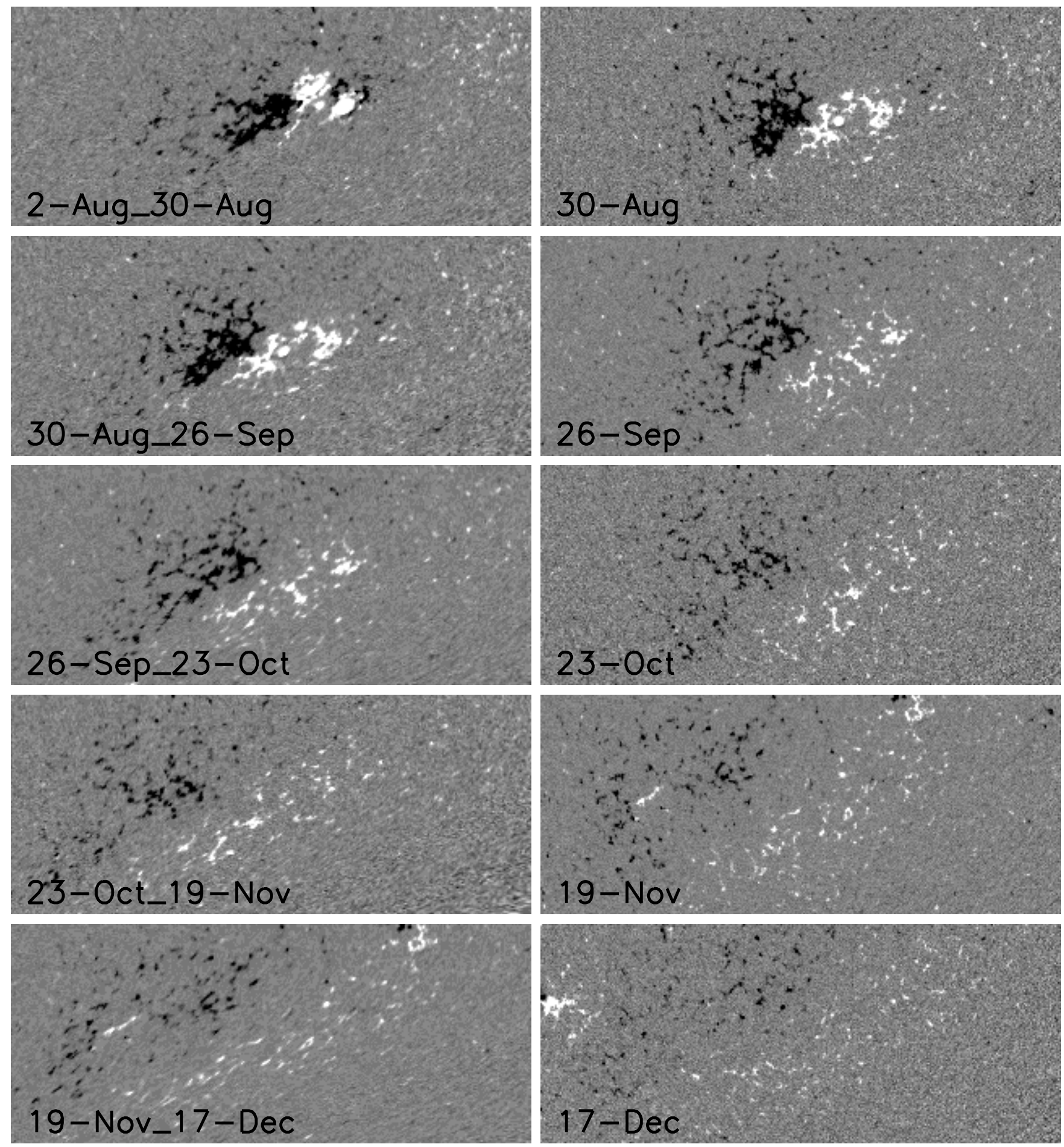

Fig. 3. On the right: SOHO/MDI magnetic maps showing the long-term evolution of AR 7978. On the left: magnetograms of the right column (first date) transformed by differential rotation after one solar rotation (second date). The difference between magnetic maps of the same line represents the evolution of the AR without the distortion of the differential rotation.

\subsection{The activity in AR 7978}

During the emergence of AR 7978, between 6-10 July 1996, the AR produced numerous flares, including an X2.6 flare and CME event on July 9 (Dryer et al. 1998). Flaring continued for the two following rotations until the disappearance of the main spots by September, 1996 (Table 1).
The rapid decrease with time of both maximum flare intensity and number of flares is remarkably different from the behavior of CME production. This indicates that, while flares and CMEs share some common physical processes (like the instability of the magnetic configuration and magnetic reconnection), they are distinct phenomena. The flare importance and the number of flares depend 
Table 1. Number of flares and CMEs from AR 7978 (see Sect. 2.4). The columns of the table give respectively: the number of the rotation, the date of central meridian passage (CMP) of the AR, the number of flares for each GOES X-ray class (from the catalog at http://www.Imsal.com/SXT/plot_goes.html), and the number of CMEs associated to AR 7978. Estimation of the CME number taking into account the data gaps (see Sect. 6.3 and Fig. 7) are given in the right column.

\begin{tabular}{|c|c|c|c|c|c|c|c|}
\hline \multirow{2}{*}{$\begin{array}{c}\text { No. } \\
\text { of rot. }\end{array}$} & \multirow{2}{*}{$\begin{array}{l}\text { first day } \\
\text { of rot. }\end{array}$} & \multicolumn{4}{|c|}{ Flares (GOES) } & \multirow{2}{*}{$\begin{array}{c}\text { CMEs } \\
\text { observed }\end{array}$} & \multirow{2}{*}{$\begin{array}{c}\text { CMEs } \\
\text { corrected }\end{array}$} \\
\hline & & $\mathrm{X}$ & M & $\mathrm{C}$ & B & & \\
\hline $1 \mathrm{st}$ & 7 July 1996 & 1 & 2 & 14 & 16 & 8 & 11 \\
\hline 2nd & 3 August 1996 & - & - & 1 & 17 & 5 & 5 \\
\hline 3rd & 30 August 1996 & - & - & - & 2 & 2 & 3 \\
\hline 4 th & 25 September 1996 & - & - & - & - & 5 & 5 \\
\hline 5 th & 23 October 1996 & - & - & - & 1 & 3 & 4 \\
\hline 6 th & 19 November 1996 & - & - & - & - & 3 & 3 \\
\hline total & & 1 & 2 & 15 & 36 & 26 & 31 \\
\hline
\end{tabular}

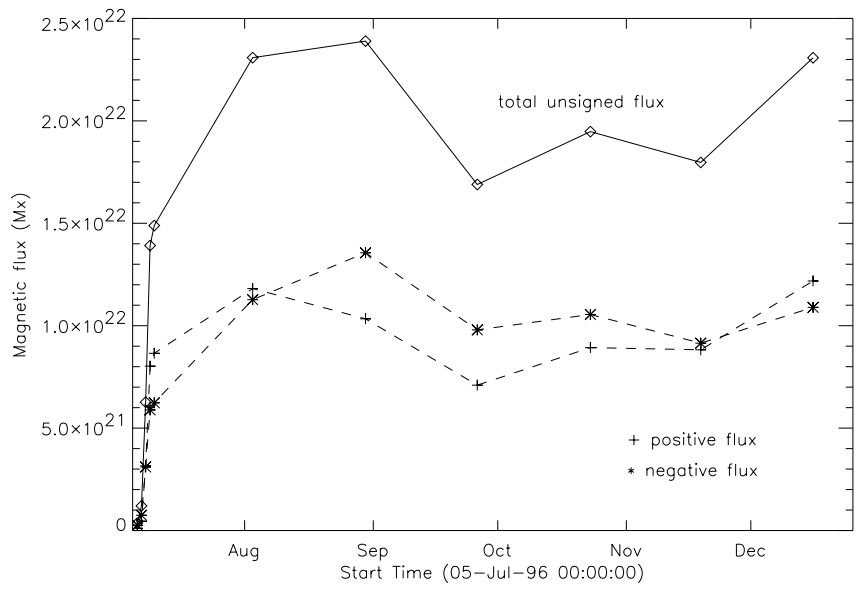

Fig. 4. Evolution of the total unsigned (continuous line), positive and negative (dashed lines) photospheric magnetic flux in AR 7978. The longitudinal magnetic field of MDI at the central meridian passages is used.

more on the free energy locally available (e.g. formation of strong current layers), while the CME production depends more on global quantities, in particular the amount of magnetic helicity (Low 1997).

CME activity, which was at first related in several cases to flare events, continued at a high level until the seventh rotation of the AR (CMP on Dec. 16, 1996). CMEs originating from AR 7978 were carefully identified in full-disc EIT images taking as proxies the presence of dimmings and/or loops in expansion. Furthermore, LASCO C2 high resolution movies were constructed in order to confirm the existence of a CME associated with the AR, and to determine its characteristics. When AR 7978 was on the far side of the Sun, we identified the related CMEs in a more subjective way, based on the position angle and the characteristics of the CME in the coronagraph observations compared to the location of the AR. This task resulted easier because of the low solar activity and the fact that, in the period from August to October 1996, AR 7978 was the only major AR on the Sun. Still, a large fraction $(\approx 2 / 3)$ of CMEs observed during this period did not originate from AR 7978; so, though this AR was alone on the Sun, we still needed a careful identification of the CME source region for each and every CME.

Table 2 lists the observed CMEs linked to AR 7978 from its appearance and until the seventh rotation. The angular size of a CME as viewed with LASCO gives an indication of the CME spatial size, but it is biased by projection effects. These include an increase of the angular size, as CMEs are launched closer to the centre disk (on both sides of the Sun), and the orientation of magnetic configuration relative to the line of sight (see Gibson \& Low 1998 for a model with a twisted flux tube viewed with different observing directions). Then, we also consider the brightness of the CME to estimate its importance. The subjective combination of the angular size and of the brightness is noted from 1 to 10 in Table 2 . It can be seen that the AR produced 15 medium to large $(>5)$ CMEs and 11 minor $(<5)$ CMEs. This classification is not critical for the helicity budget, but it is rather a detail whether to take into account, or not, the "smaller" CMEs in the budget.

For future space weather studies, we add the following complementary information. Most of the CMEs which originated from AR 7978 were not geoeffective. However, comparing our CME list with data corresponding to the geomagnetic index Dst (see ftp://ftp.ngdc.noaa.gov/STP/GEOMAGNETIC.DATA/INDICES/DST/), whose perturbation can be indicative of a geomagnetic storm (González et al. 1994, 1999), we have found that a very intense $\left(D s t_{\min }<-100 n T\right)$ geomagnetic storm occurred four days after the CME on 19 October, 1996. This CME was associated with the disparition brusque of a filament as shown in $\mathrm{H} \alpha$ images obtained at Meudon Observatory. Figure 6 shows the evolution in LASCO $\mathrm{C} 2$ of this CME. A similar CME eruption linked to this AR has been studied by van Driel-Gesztelyi et al. (1998). 
Table 2. The CMEs ejected by AR 7978. The first column indicates the date and time of the first appearance of the ejection in LASCO C2. Times in parentheses indicate different C2 commencement for the CME given in the CUA list than given in the GSFC list (see below). The second twin columns give the maximum angular extension of the CME (projected in the plane of sky); two values are given coming from the two independent catalogs prepared by C. St Cyr (GFSC) and by S. Yashiro \& G. Michalek (Catholic University of America). When we found no values in these catalogs, the angular extension come from the authors (in parentheses). In the third column we classify the CMEs according to their size, projected in the plane of the sky, from 1 to 10, such that growing numbers imply growing importance (see Sect. 2.4). The fourth column indicates with $\mathrm{H}$ the halo CMEs and with $\mathrm{T}$ the large-scale transequatorial events. In the last column we indicate the position of the AR at the time of the CME; rotation phase 0 indicates that the AR is at CMP on the first rotation, 0.25 that it is at the West limb and so on. The rotation phase numbers increase as the AR goes back to the CMP again and again.

\begin{tabular}{|c|c|c|c|c|c|}
\hline \multirow[t]{2}{*}{ Date } & \multicolumn{2}{|c|}{ angular size } & \multirow[t]{2}{*}{ Importance } & \multirow[t]{2}{*}{ Remarks } & \multirow[t]{2}{*}{ Rotation phase } \\
\hline & St Cyr & Yashiro & & & \\
\hline $1996 / 07 / 0806: 28$ & 40 & 35 & 6 & & 0.04 \\
\hline 1996/07/09 12:28 & 80 & 86 & 7 & $\mathrm{~T}$ & 0.08 \\
\hline 1996/07/10 07:28 & 29 & 35 & 4 & & 0.11 \\
\hline 1996/07/12 15:37 & 40 & 68 & 4 & & 0.20 \\
\hline 1996/07/14 15:17 & 32 & 50 & 3 & & 0.28 \\
\hline 1996/07/15 04:35 (10:46) & 14 & 43 & 2 & & 0.30 \\
\hline 1996/07/16 05:12 (08:27) & 29 & 47 & 4 & & 0.33 \\
\hline 1996/07/17 05:20 & 20 & 34 & 6 & & 0.37 \\
\hline 1996/08/13 16:09 & 138 & 153 & 7 & & 1.40 \\
\hline 1996/08/14 19:30 & 300 & 235 & 2 & & 1.44 \\
\hline 1996/08/16 14:14 & 360 & & 9 & $\mathrm{H}$ & 1.50 \\
\hline 1996/08/19 14:09 (11:18) & 63 & 82 & 9 & & 1.61 \\
\hline 1996/08/22 08:38 & 110 & 125 & 9 & $\mathrm{~T}$ & 1.72 \\
\hline 1996/09/03 03:25 & $(20)$ & & 6 & & 2.14 \\
\hline 1996/09/04 00:43 & 35 & & 4 & & 2.18 \\
\hline 1996/09/25 23:00 & 140 & & 9 & & 3.00 \\
\hline 1996/10/05 09:45 (09:37) & 253 & 161 & 9 & $\mathrm{~T}$ & 3.35 \\
\hline 1996/10/18 07:00 & $(20)$ & & 2 & & 3.83 \\
\hline 1996/10/19 15:00 (17:39) & 192 & 206 & 10 & & 3.88 \\
\hline 1996/10/20 19:03 & $(26)$ & & 7 & & 3.92 \\
\hline 1996/10/31 07:00 (06:25) & 13 & & 2 & & 4.30 \\
\hline 1996/11/07 09:00 (07:25) & 22 & 36 & 3 & & 4.56 \\
\hline 1996/11/07 19:05 (23:20) & 360 & & 10 & $\mathrm{H}$ & 4.58 \\
\hline $1996 / 11 / 24$ 17:40 (17:52) & 57 & 77 & 4 & & 5.22 \\
\hline $1996 / 11 / 2616: 57(15: 35)$ & 39 & 41 & 10 & $\mathrm{~T}$ & 5.29 \\
\hline $1996 / 11 / 28$ 17:00 (16:50) & 79 & 78 & 9 & $\mathrm{~T}$ & 5.37 \\
\hline
\end{tabular}

\subsection{Evolution of the computed coronal magnetic field}

Since only the longitudinal component of $\boldsymbol{B}$ is available from MDI data, the coronal field is computed under the linear (or constant $\alpha$ ) force-free field assumption:

$$
\nabla \times \boldsymbol{B}=\alpha \boldsymbol{B},
$$

using a fast Fourier transform method (Alissandrakis 1981). The vertical (or normal to the photosphere) component $B_{n}$ of the field is written as:

$B_{n}=\sum_{n_{x}=1}^{N_{x}} \sum_{n_{y}=1}^{N_{y}} \widetilde{B}_{n_{x}, n_{y}} \exp (-l z)$, where $x, y$ are the Cartesian horizontal coordinates, $z$ is the vertical coordinate, $\widetilde{B}_{n_{x}, n_{y}}$ is the Fourier amplitude of the harmonic $\left(n_{x}, n_{y}\right), l=\sqrt{k_{x}^{2}+k_{y}^{2}-\alpha^{2}}, k_{x}=$ $2 \pi n_{x} / L, k_{y}=2 \pi n_{y} / L$, being $L$ the horizontal extension of the computational box. The horizontal components $\left(B_{x}, B_{y}\right)$ of the field are expressed in the same way as $B_{n}$ in Eq. (2) (Alissandrakis 1981). The Fourier amplitudes $\widetilde{B}_{n_{x}, n_{y}}$ could be computed from the observed longitudinal magnetic field values at the photosphere, but our experience shows that it is better to estimate $B_{n}$ from the measured longitudinal component (the magnetic field at the photosphere is more vertically oriented than force free). A better model of the coronal field would be 

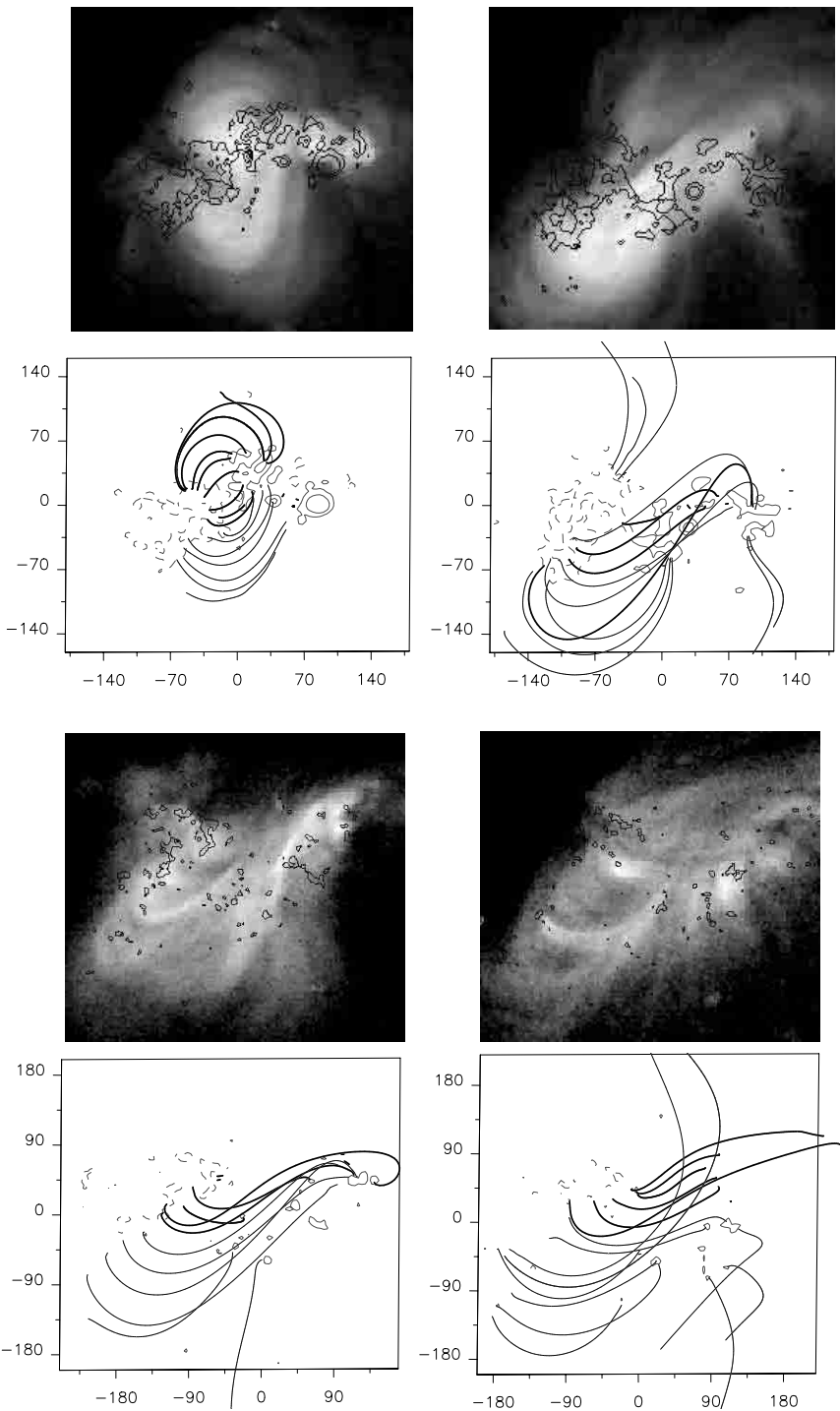

Fig. 5. Yohkoh/SXT observations and linear force-free computations of the coronal field using SOHO/MDI magnetic maps. The top rows correspond to Aug. 3 and 30, while bottom rows to Sep. 25 and Oct. 23, respectively. The $\alpha$ value is selected to have the best global match between the SXT loops and the computed field lines (see Sect. 2.5). We have drawn with thick (thin) continuous lines the field lines computed taking the highest (lowest) value of $\alpha$, and have represented them on the same figure (see values in Table 3 ). The abscissas and ordinates in each map are expressed in Mm. Isocontours $( \pm 100$, $500,1000 \mathrm{G}$ ) of the line of sight magnetic field have been drawn with continuous/dashed lines for positive/negative values.

obtained by computing $B_{n}$ from the three measured components of the photospheric field, but presently the large noise $(\approx 200 \mathrm{G})$ in the transverse field data precludes such approach. The value of $\alpha$ in Eq. (1) is determined through an iterative process. First, we compute the coronal field assuming a given value for $\alpha$, then we compare the computed field lines to the observed SXT coronal loops and, finally, through successive steps we select the value of $\alpha$ that gives the best global fit. We want to point out that, in order to better fit the observed SXT loops in the southern
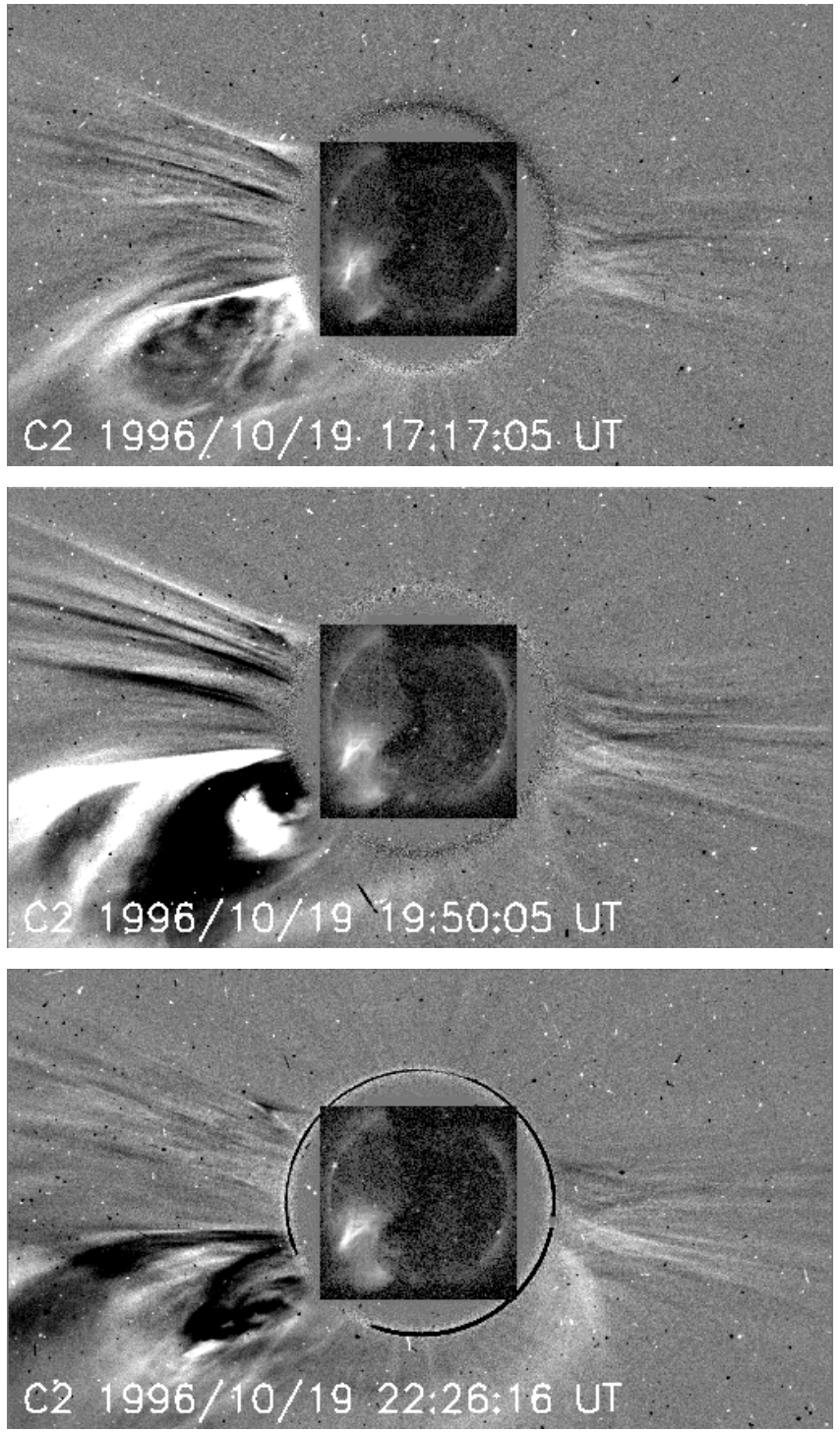

Fig. 6. An example of a CME which originated from AR 7978 as observed with LASCO C2. Running difference images show the evolution of the CME on October 19, 1996. Yohkoh/SXT images, taken with time difference of a few $(\leq 10)$ min. to the times of the $\mathrm{C} 2$ observations, show the lower coronal evolution during the CME. Note the formation of a cusp structure in AR 7978.

portion of the AR during the second rotation, the height of computed magnetic field lines has been multiplied by a factor 1.5 .

We have found that not all SXT loops observed at a given time can be represented using the same value of $\alpha$. In general, a shear gradient exists in the North-South direction. Figure 5 illustrates the temporal evolution of the coronal shear from the second to the fifth rotation and, at the same time, the just mentioned shear gradient. In Table 3 we have listed the maximum and minimum values of $\alpha$ determined for each rotation; these values correspond to the North and South portion of the AR, respectively. It can be seen that $\alpha$ increases from the first to the fourth rotation, after that it starts decreasing. 
Table 3. Force-free parameter $\alpha$ for AR 7978 along six rotations. Estimation of $\alpha$ from the best matching between SXT loops and the computation of the coronal magnetic field from MDI magnetograms. Two values of $\alpha$ are given per rotation because a gradient of shear is present in the SXT loops between the North and South part of the AR (see Sect. 2.5 and Fig. 5). All values are in units of $10^{-2} \mathrm{Mm}^{-1}$.

\begin{tabular}{lcc}
\hline Date & $\alpha$ - South & $\alpha-$ North \\
\hline 7 July, 1996 & - & 1.00 \\
3 August, 1996 & 0.30 & 0.75 \\
30 August, 1996 & 0.90 & 1.00 \\
25 September, 1996 & 1.00 & 1.40 \\
23 October, 1996 & 0.90 & 1.40 \\
18 November, 1996 & 0.90 & 0.90 \\
\hline
\end{tabular}

\section{Magnetic helicity}

\subsection{Definition of the relative magnetic helicity}

The magnetic helicity of a field $\boldsymbol{B}$ within a volume $V$ is defined by:

$H=\int_{V} \boldsymbol{A} \cdot \boldsymbol{B} \mathrm{d} V$

where the vector potential $\boldsymbol{A}$ satisfies

$B=\nabla \times A$.

However, Eq. (3) is physically meaningful only when the magnetic field is fully contained inside $V$ (i.e. at any point of the surface $S$ surrounding $V$, the normal component $B_{n}=\boldsymbol{B} . \hat{n}$ vanishes); this is so because the vector potential is defined through a gauge transformation $\left(\boldsymbol{A}^{\prime}=\boldsymbol{A}+\boldsymbol{\nabla} \Phi\right)$, then $H$ is gauge-invariant only when $B_{n}=0$.

Berger \& Fields (1984) have shown that for cases where $B_{n} \neq 0$ one can define a relative magnetic helicity $\left(H_{\mathrm{r}}\right)$ subtracting the helicity of a reference field $\boldsymbol{B}_{\mathbf{0}}$, having the same distribution of $B_{n}$ on $S$ :

$H_{\mathrm{r}}=\int_{V} \boldsymbol{A} \cdot \boldsymbol{B} \mathrm{d} V-\int_{V} \boldsymbol{A}_{\mathbf{0}} \cdot \boldsymbol{B}_{\mathbf{0}} \mathrm{d} V$,

Berger \& Fields (1984) and Finn \& Antonsen (1985) have shown that $H_{\mathrm{r}}$ is gauge-invariant, and that $H_{\mathrm{r}}$ does not depend on the common extension of $\boldsymbol{B}$ and $\boldsymbol{B}_{\mathbf{0}}$ outside $V$. A convenient choice for the reference field $\boldsymbol{B}_{\mathbf{0}}$ is a potential field with $\boldsymbol{A}_{\mathbf{0}}$ satisfying Eq. (4), $\boldsymbol{\nabla} \cdot \boldsymbol{A}_{\mathbf{0}}=0$ and $\left(A_{0}\right)_{n}=0$ on $S$; in this way the helicity $\int_{V} \boldsymbol{A}_{\mathbf{0}} \cdot \boldsymbol{B}_{\mathbf{0}} \mathrm{d} V$ vanishes (Berger 1988). Furthermore, imposing on $S$ the boundary condition:

$\hat{n} \times \boldsymbol{A}=\hat{n} \times \boldsymbol{A}_{\mathbf{0}}$

(that is to say, setting the transverse components of $\boldsymbol{A}$ to those of $\boldsymbol{A}_{\mathbf{0}}$, a choice that keeps the same distribution of $B_{n}$ on $S$ ), the relative helicity $H_{\mathrm{r}}$ (Eq. (5)) reduces to the initial expression given for fields confined inside $V$
(Eq. (3)). It is worth noticing that DeVore (2000) has extended the work of Berger (1988) to any other gauge for $\boldsymbol{A}$ provided the boundary condition expressed by Eq. (6) is used.

\subsection{Computation of the relative magnetic helicity}

Magnetic fields are present in three main regions having very different physical characteristics: the convective zone, the corona and the interplanetary medium. In the convective zone the magnetic field is present only in a tiny portion of the volume, probably in the form of discrete twisted flux tubes with a strong field $\left(\approx 10^{5} \mathrm{G}\right.$ at the base of the convective zone). After emergence in the corona, the magnetic field fills the available space (the plasma $\beta$ parameter, which is the ratio between plasma and magnetic pressure, is low in the corona). The strength of the field decreases to $\approx 100 \mathrm{G}$. After ejection of part of this field in the form of a CME, the corresponding magnetic field is observed in the interplanetary medium as a magnetic cloud moving outwards typically at about the solar wind speed. The expansion of the flux tube is so large that the field strength is only $\approx 10^{-4} \mathrm{G}$ at $1 \mathrm{AU}$.

Because of the very different physical conditions present in these three regions, a different procedure is required to estimate the gauge-independent magnetic helicity $H_{\mathrm{r}}$ in each of them: for twisted flux tubes in the convection zone see Appendix A, for the corona see Sect. 5 and for magnetic clouds see Sect. 6. Magnetic helicity is also transferred by plasma flows from one region to a neighbor one. We analyze in the next section the transfer from the convective zone to the corona.

\section{Injection of magnetic helicity}

\subsection{General expressions}

Since $H_{\mathrm{r}}$ is well preserved under solar conditions, the only way helicity can be modified inside $V$ is because of helicity flux crossing the boundary $S$. The change of relative helicity is written as (Berger \& Fields 1984):

$\frac{\mathrm{d} H_{\mathrm{r}}}{\mathrm{d} t}=-2 \int_{S}\left[\left(\boldsymbol{A}_{\mathbf{0}} \cdot \boldsymbol{v}\right) \boldsymbol{B}-\left(\boldsymbol{A}_{\mathbf{0}} \cdot \boldsymbol{B}\right) \boldsymbol{v}\right] . \mathrm{d} \boldsymbol{S}$,

where $\boldsymbol{v}$ is the velocity of the plasma. The last term on the right hand side of Eq. (7) represents a direct "inflow" of plasma (for $\boldsymbol{v} . \mathrm{d} \boldsymbol{S}>0$, otherwise "inflow" should be replaced by "outflow"), which carries magnetic helicity together with the magnetic flux. In the case studied below, where $S$ is at the photospheric level, this term is difficult to evaluate because there is no information about the magnetic field below the photosphere. Therefore, we will consider only the first term in the right-hand side of Eq. (7). This term represents the injection of helicity by plasma motions parallel to the surface $S$. In this case, Eq. (7) reduces to:

$\frac{\mathrm{d} H_{\mathrm{r}}}{\mathrm{d} t}=-2 \int_{S}\left(\boldsymbol{A}_{\mathbf{0}} \cdot \boldsymbol{v}\right) B_{n} \mathrm{~d} S$. 
Table 4. The magnetic helicity budget of AR 7978 is listed per rotation. An interval of helicity is given for the corona (see Sect. 5.2) and for the cloud estimations with the observed number of CMEs (considering the two limits, 0.5 and 2 AU, for the length of magnetic clouds: see Sect. 6). Correcting from the LASCO data gaps (Fig. 7) is likely to increase $\Delta H_{\text {mag.clouds }}$ only up to a factor 1.2 (Sect. 6.3). Two values of the helicity injected by the differential rotation are given for the first rotation: for AR 7978 alone and, in parenthesis, for its neighbor decaying ARs (see Fig. 1). The budget of the right column is discussed in Sect. 7. All values are in units of $10^{42} \mathrm{Mx}^{2}$.

\begin{tabular}{|c|c|c|c|c|c|}
\hline No. of rot. & Date & $H_{\text {corona }}$ & $\Delta H_{\text {diff.rot. }}$ & $\Delta H_{\text {mag.clouds }}$ & $\Delta H_{\text {corona }}-\Delta H_{\text {diff.rot. }}$ \\
\hline & 7 Jul. & - & & & \\
\hline \multirow[t]{2}{*}{1 st } & & & $0.2(2)$ & {$[16,64]$} & $(\approx 7)$ \\
\hline & 3 Aug. & {$[5,11]$} & & & \\
\hline \multirow[t]{2}{*}{ 2nd } & & & 3. & {$[10,40]$} & 9 \\
\hline & 30 Aug. & {$[17,23]$} & & & \\
\hline \multirow[t]{2}{*}{$3 r d$} & & & 3. & {$[4,16]$} & -13 \\
\hline & 25 Sep. & {$[9,12]$} & & & \\
\hline \multirow[t]{2}{*}{4 th } & & & 1. & {$[10,40]$} & -7 \\
\hline & 23 Oct. & {$[4,6]$} & & & \\
\hline \multirow[t]{2}{*}{5 th } & & & 0.8 & {$[6,24]$} & -2 \\
\hline & 19 Nov. & (4) & & & \\
\hline 6 th & & & 0.3 & {$[6,24]$} & - \\
\hline total & & - & 8.3 & {$[52,208]$} & - \\
\hline
\end{tabular}

This helicity injection depends only on plasma motions $\boldsymbol{v}$ and the normal field $B_{n}$. This is because $\boldsymbol{A}_{\mathbf{0}}$ for the potential field $\boldsymbol{B}_{\mathbf{0}}$ can be expressed only in function of $B_{n}$ on $S$. This contrasts with the injection of energy (Poynting flux), which also depends on the stress of the field in the corona. In this way, we can compute the injection of magnetic helicity without solving the magnetic field equations in the corona.

For simplicity, let's assume that the photosphere is locally planar at the scale size of an AR (see Berger \& Ruzmaikin 2000 for a spherical version). Computing $\boldsymbol{A}_{\mathbf{0}}$ as a function of the $B_{n}$ distribution, Berger $(1984,1988)$ derived an expression for $\mathrm{d} H_{\mathrm{r}} / \mathrm{d} t$ that depends only on observable photospheric quantities $\left(B_{n}\right.$ and $\left.\boldsymbol{v}\right)$ :

$$
\frac{\mathrm{d} H_{\mathrm{r}}}{\mathrm{d} t}=-\left.\frac{1}{\pi} \int_{S} \int_{S} \frac{\boldsymbol{R} \times \boldsymbol{v}(\boldsymbol{r})}{R^{2}}\right|_{n} B_{n}(\boldsymbol{r}) B_{n}\left(\boldsymbol{r}^{\prime}\right) \mathrm{d} S . \mathrm{d} S^{\prime},
$$

where $\boldsymbol{R}=\boldsymbol{r}-\boldsymbol{r}^{\prime}$ is the difference in spatial positions on the photospheric plane. This equation involves a double integration (on $\boldsymbol{r}$ and $\boldsymbol{r}^{\prime}$ ) on the magnetogram. Let us define $\theta$ as the angle between $\boldsymbol{R}$ and a fixed direction (e.g. the East-West direction) with trigonometric convention (counterclockwise rotation is positive), then Eq. (9) is transformed to (Berger 1986):

$$
\frac{\mathrm{d} H_{\mathrm{r}}}{\mathrm{d} t}=-\frac{1}{2 \pi} \int_{S} \int_{S} \frac{\mathrm{d} \theta}{\mathrm{d} t} B_{n}(\boldsymbol{r}) B_{n}\left(\boldsymbol{r}^{\prime}\right) \mathrm{d} S . \mathrm{d} S^{\prime} .
$$

This equation shows that the helicity injection rate can be understood as the summation of the rotation rate of all the pairs of elementary fluxes weighted with their magnetic flux.

Considering only horizontal motions, the elementary fluxes $B_{n}(\boldsymbol{r})$.d $S$ are time-independent (though they can still change in shape or field strength) and Eq. (10) can be integrated:

$\Delta H_{\mathrm{r}}(t)=-\frac{1}{2 \pi} \int_{S} \int_{S} \Delta \theta(\boldsymbol{R}, t) B_{n}(\boldsymbol{r}) B_{n}\left(\boldsymbol{r}^{\prime}\right) \mathrm{d} S . \mathrm{d} S^{\prime}$,

where $\Delta H_{\mathrm{r}}$ and $\Delta \theta$ are respectively the variation of $H_{\mathrm{r}}$ and $\theta$. As in the case of Eqs. (10), (11) gives a geometrical interpretation to the injected helicity. Démoulin et al. (2001) applied Eq. (11) to shear velocity profiles and to theoretical bipolar magnetic configurations. They found that Eq. (11) can be separated in two terms: twist and writhe. For differential rotation, twist and writhe helicity have opposite signs and partially cancel. This implies that the evolution with time of the helicity is generally nonmonotonous and that differential rotation is a relatively inefficient way to bring magnetic helicity in the corona. As shown below, such results are also found for the magnetic configuration of AR 7978.

\subsection{The helicity injected in AR 7978}

The magnetic helicity injected in AR 7978 per solar rotation, as computed from Eq. (11), is summarized in the fourth column of Table 4 . These values have been obtained considering as initial condition the MDI magnetogram of the AR obtained at CMP rotation per rotation (Fig. 3). This initial configuration is evolved applying differential rotation along one Carrington rotation, and the integrated magnetic helicity over that period of time is listed. As shown by Démoulin et al. (2001), the generation rate of helicity is larger when the bipole tilt is small (see the values for the second and third rotations). This injection declines when the bipole has a tilt closer to $45^{\circ}$ (Oct. and 
Nov. rotations). On Aug. 3 and Aug. 30 rotations, the injection rate $\left(\approx 10^{41} \mathrm{Mx}^{2} /\right.$ day $)$ corresponds typically to the injection rate found for a dipole with a magnetic flux $\Phi$ of $10^{22} \mathrm{Mx}$ (which is approximately the flux in AR 7978, see Fig. 4). This value is only one third of the one estimated by DeVore (2000) because AR 7978 is at a latitude of $-10^{\circ}$, while the dipole in DeVore's work is located at $30^{\circ}$ latitude.

The total helicity injected into AR 7978 by differential rotation is $\approx 8.3 \times 10^{42} \mathrm{Mx}^{2}$. This value is in close agreement with the estimate of the maximum total helicity injected in a dipole that is initially parallel to the equator $\left(10^{43} \mathrm{Mx}^{2}\right)$ having a magnetic flux $\Phi$ of $\approx 10^{22} \mathrm{Mx}$ (see Fig. 5 in Démoulin et al. 2001). In fact, AR 7978 keeps its magnetic coherence long enough so that differential rotation has nearly the time to reach the maximum injection of helicity; $\mathrm{d} H_{\mathrm{r}} / \mathrm{d} t$ is one order of magnitude smaller during the 6th (Nov.) rotation compared to its value during the 2 nd and 3th rotations. These results are in agreement with DeVore (2000).

\section{Magnetic helicity in the corona}

\subsection{Method}

The coronal magnetic field is computed using a linear force-free approach where the observed magnetograms are used as boundary conditions, see Sect. 2.5. The large spatial extension of the AR and the complexity of the observed magnetic polarities implies that a large number of harmonics should be considered in both horizontal directions $\left(N_{x}=N_{y}=256\right.$ in this particular case). Following the results by Berger (1985), the relative magnetic helicity is:

$H_{\mathrm{r}}=2 \alpha \sum_{n_{x}=1}^{N_{x}} \sum_{n_{y}=1}^{N_{y}} \frac{\left|\widetilde{B}_{n_{x}, n_{y}}^{2}\right|}{l\left(k_{x}^{2}+k_{y}^{2}\right)}$

where, as in Sect. 2.5, $\widetilde{B}_{n_{x}, n_{y}}$ is the Fourier amplitude, $l=\sqrt{k_{x}^{2}+k_{y}^{2}-\alpha^{2}}, k_{x}=2 \pi n_{x} / L, k_{y}=2 \pi n_{y} / L$ with $L$ being the horizontal extension of the computational box.

For small $\alpha$ values $\left(\ll 2 \pi / L\right.$, so $\left.l \approx \sqrt{k_{x}^{2}+k_{y}^{2}}\right)$, Eq. (12) shows that $H_{\mathrm{r}}$ grows proportionally with $\alpha$ for a given magnetogram. As $\alpha$ becomes very close to $2 \pi / L$, the factor $l$ at the denominator decreases (this effect being strongest for harmonics $(1,0)$ and $(0,1))$; then, the helicity increases rapidly (see Fig. 1 in Berger 1985, though this figure corresponds to a spherical geometry the effect is the same in Cartesian geometry). In the limit $\alpha \rightarrow 2 \pi / L$, the helicity is infinite. Therefore, when the value of $\alpha$ used in the model of the coronal field is close to $2 \pi / L$, the computed helicity can be an overestimation of the real helicity value. A way to avoid this is to use a linearized form for $H_{\mathrm{r}}$ :

$H_{\mathrm{r}, \text { lin }}=2 \alpha \sum_{n_{x}=1}^{N_{x}} \sum_{n_{y}=1}^{N_{y}} \frac{\left|\widetilde{B}_{n_{x}, n_{y}}^{2}\right|}{\left(k_{x}^{2}+k_{y}^{2}\right)^{3 / 2}}$.
This provides an estimate on the minimum helicity present in the coronal field.

Apart from computing helicity values within an interval (see Sect. 5.2), it is difficult to estimate an error bar for the computed helicity because the observed coronal field is not fully relaxed to a linear force-free field. The general property of a linear force-free field is to make the shorter (resp. longer) field lines less (resp. more) sheared than the observed coronal loops. This implies that the linear forcefree field has very low (resp. too large) magnetic helicity in the small scales (resp. large scales) compared to the coronal field (see e.g. Schmieder et al. 1996). This property is rooted in the inverse cascade (towards the large spatial scales) of magnetic helicity which occurs when a magnetic field relaxes to a linear force-free field (minimizing the magnetic energy under the constraint of a fixed total relative helicity). Presently, one can only suppose that the over/under estimate of helicity at large/small scales compensates well enough to keep the right order of magnitude because the value of $\alpha$ is selected so that the linear force-free field best represent the observed coronal loops.

\subsection{The coronal helicity of $A R 7978$}

AR 7978 emerged in a non-potential state with the helicity sign corresponding to the southern hemisphere (Pevtsov et al. 1995). The AR appeared very close to a pre-existing bipole having helicity of the opposite sign (van Driel-Gesztelyi et al. 1999; Mandrini et al. 2000).

We have computed the coronal $H_{\mathrm{r}}$ of AR 7978 for the magnetic field models obtained from MDI magnetograms and shown in Fig. 5. The calculation has been done considering a computational box centered in the AR and having the same extension in all cases. As shown in Fig. 5 and Table 3, the value of $\alpha$ varies along the AR (from North to South), and also from rotation to rotation. In the third column of Table 4 we list an interval for $H_{\mathrm{r}}$ per rotation, where the minimum (resp. maximum) values have been obtained from the minimum (resp. maximum) values of $\alpha$ in each rotation in the linearized approach (Eq. (13)). Therefore, these values are likely to be a lower bound in the estimation of the coronal $H_{\mathrm{r}}$. However, we want to stress that in those cases where $\alpha$ was close to $2 \pi / L$, the $H_{\mathrm{r}}$ computed from Eq. (12) was at most a factor $\approx 1.5$ larger than the linearized value computed from Eq. (13). The value between parenthesis for Nov. 18 rotation is only indicative. It was obtained for the coronal field model of the southern portion of AR 7978, the SXT loops in the northern portion were so faint that it was not possible to model them.

In order to estimate the degree of confidence one can have in the $H_{\mathrm{r}}$ given by the linear force-free field, we have done the following test. On August 30 rotation, AR 7978 has such a simple magnetic configuration that applying a hypothetic shearing motion parallel to its inversion line, and opposite to the observed shear, we can bring it to an approximately potential configuration. Doing so, we can 
estimate the amount of helicity coming from this shearing motion; we have found that this last value is similar to the $H_{\mathrm{r}}$ computed from the linear force-free field.

Can the helicity injected in AR 7978 by differential rotation be the origin of the estimated coronal helicity? On Aug. 3 rotation the value of the minimum coronal helicity, $\approx 5 \times 10^{42} \mathrm{Mx}^{2}$ (Table 4 ), is still higher than the maximum value $\left(\approx 2 \times 10^{42} \mathrm{Mx}^{2}\right)$ given by differential rotation acting on AR 7978 and its neighbor decaying ARs (from July 7 to Aug. 3). When we restrict the computation of the injected helicity only to AR 7978 the obtained result is one order of magnitude smaller. Later on, the minimum coronal helicity is $\approx 17 \times 10^{42} \mathrm{Mx}^{2}$ on Aug. 30, increasing in $\approx 12 \times 10^{42} \mathrm{Mx}^{2}$ compared with Aug. 3, while the injection by differential rotation during that period is only of $\approx 3 \times 10^{42} \mathrm{Mx}^{2}$, so a factor 4 smaller. If we rather consider for the variation of the coronal helicity the average between the minimum and maximum values (see Table 4), we find comparing the same two cases (July 7 to Aug. 3 and Aug. 3 to Aug. 30) that the helicity injected by differential rotation is always a factor 4 lower. After that, the coronal helicity decreases, but we have also to consider the helicity ejected from the Sun (see Sect. 6) when analyzing the helicity budget of the AR.

All these results indicate that differential rotation does not inject enough magnetic helicity into the coronal field to account for the helicity found there, even if we take the minimum of the estimates for the coronal helicity.

\section{Magnetic helicity of interplanetary clouds}

\subsection{Method}

A series of studies, related to the magnetic field in interplanetary clouds, has shown that they are basically twisted flux tubes expanding and moving outwards in the interplanetary medium. Moreover, "in situ" measurements of the magnetic field are globally well-described by a linear-force free field, suggesting that Taylor's relaxation (1974) was efficient after their launch from the corona (Lepping et al. 1990, 1997; Marubashi 1997; Osherovich \& Burlaga 1997; Shimazu \& Marubashi 2000). Indeed, only one harmonic of the linear force-free field in cylindrical geometry is enough to describe these measurements of $\boldsymbol{B}$ ! This clearly contrasts with the large number of harmonics needed to compute the coronal field. The magnetic field of interplanetary clouds, in general, is well-described in cylindrical coordinates by:

$\boldsymbol{B}=B_{\mathrm{o}}\left[J_{1}(\alpha r) \hat{u}_{\varphi}+J_{0}(\alpha r) \hat{u}_{z}\right]$

where $J_{n}$ is the Bessel function of the first kind of order $n$, $B_{\mathrm{O}}$ is the strength of the field and $\alpha=\nabla \times \boldsymbol{B} / \boldsymbol{B}$. The radius $R$ of the magnetic cloud is defined by the first zero of $B_{z}$ in Eq. (14), so $J_{0}(\alpha R)=0$. Using the representation in series of Bessel functions, Eq. (4) is solved for the vector potential:

$$
\boldsymbol{A}=\boldsymbol{B} / \alpha
$$

Computing the helicity directly from $\int_{V} \boldsymbol{A} \cdot \boldsymbol{B} \mathrm{d} V=$ $\frac{2 \pi B_{0}^{2}}{\alpha} \Delta z \int_{0}^{R}\left[J_{1}^{2}(\alpha r)+J_{0}^{2}(\alpha r)\right] r \mathrm{~d} r$ gives the good numerical answer for the relative helicity (because $\int_{0}^{R} J_{1}^{2}(\alpha r) r \mathrm{~d} r=$ $\left.\int_{0}^{R} J_{0}^{2}(\alpha r) r \mathrm{~d} r\right)$, but following Berger (1999) this is formally incorrect (see Appendix A). Following Eq. (A.4) the relative helicity present per unit length in the twisted flux tube is:

$\frac{\mathrm{d} H_{\mathrm{r}}}{\mathrm{d} z}=2 \int_{0}^{R} A_{\varphi} B_{\varphi} 2 \pi r \mathrm{~d} r=\frac{4 \pi B_{\mathrm{o}}^{2}}{\alpha} \int_{0}^{R} J_{1}^{2}(\alpha r) r \mathrm{~d} r$.

A numerical integration of this equation gives:

$\frac{\mathrm{d} H_{\mathrm{r}}}{\mathrm{d} z} \approx 0.70 B_{\mathrm{o}}^{2} R^{3}$.

This is slightly different from the result given by DeVore (2000), where the numerical factor is 0.60 . The difference with our results is linked to the analytic evaluation of the integrals by DeVore; that is to say, his steps from Eqs. (47) to (48). The flux of $B_{z}$ is $\Phi=2 \pi \int_{0}^{R} B_{z} r \mathrm{~d} r \approx$ $1.4 B_{0} R^{2}$, in agreement with DeVore (2000). Finally, the relative helicity per unit length is:

$\frac{\mathrm{d} H_{\mathrm{r}}}{\mathrm{d} z} \approx \frac{0.4}{R} \Phi^{2}$

\subsection{The helicity ejected from AR 7978}

Since we have no way to estimate the helicity in a CME, we can only make an association between the number of CMEs ejected by AR 7978 and the magnetic clouds originated by them assuming a one to one correspondence (as DeVore 2000 did). It is a strong hypothesis to suppose that each CME detected by LASCO becomes a magnetic cloud but there are increasing evidences that it is so (Webb et al. 2000). Because of the lack of quantitative data on the magnetic clouds linked to AR 7978, we rather rely below on another set of well observed and modeled magnetic clouds. The magnetic field has been measured in a set of 18 well-observed interplanetary clouds and a close fit to the observations has been obtained using one harmonic of a cylindrical linear force-free field by Lepping et al. (1990; see also Burlaga et al. 1998; Lepping et al. 1991, 1997 for individual examples). Taking the average value of $B_{\mathrm{o}}\left(2 \times 10^{-4} \mathrm{G}\right)$ and $R\left(2 \times 10^{12} \mathrm{~cm}\right)$ from the largest set of analyzed cases Lepping et al. (1990) we can compute the magnetic helicity per unit of length in a cloud (Eq. (18)). Following DeVore (2000), the length of the flux tube was estimated as $\approx 0.5 \mathrm{AU}$ for clouds observed at $1 \mathrm{AU}$ from the Sun. This gives an average helicity estimate of $\approx 2 \times 10^{42} \mathrm{Mx}^{2}$ per magnetic cloud, so per CME.

First, let us consider the evolution from the second to the sixth rotation when AR 7978 was dipole-like and nearly alone on the Sun. AR 7978 ejected at least 18 CMEs during that period (see Table 2). Multiplying that number of CMEs by the above estimate of $\approx 2 \times 10^{42} \mathrm{Mx}^{2}$ per $\mathrm{CME}$, we find that a magnetic helicity $\approx 36 \times 10^{42} \mathrm{Mx}^{2}$ was ejected from AR 7978 from the second to the sixth rotation while differential rotation only produced $\approx 8.1 \times 10^{42} \mathrm{Mx}^{2}$ 
(see Table 4). Then, the magnetic helicity ejected is a factor 4 larger than the helicity injected by differential rotation.

We have also computed the injection of helicity for AR 7978 and its surrounding during the 1st (July) rotation (Table 4). Including this rotation, we see that the deficit of helicity injected by differential rotation is still higher compared to the estimated ejected helicity (at most $\approx 10.1 \times 10^{42} \mathrm{Mx}^{2}$ compared to $\approx 52 \times 10^{42} \mathrm{Mx}^{2}$ ejected for $26 \mathrm{CMEs}$ ). So we get at least a factor 5 when we consider their total budgets over the full AR lifetime.

\subsection{Uncertainties on the helicity ejected}

The magnetic helicity ejected is subjected to several uncertainties. First, probably not all CMEs associated with AR 7978 were observed, second we assume that the average CME helicity corresponds to the average helicity of a magnetic cloud set, and third the length of a magnetic cloud is probably much larger than $0.5 \mathrm{AU}$ for clouds observed at 1 AU away from the Sun as discussed below.

\subsubsection{CME count}

First we estimate the number of CMEs which were missed by LASCO. We are, a priori, expecting to miss some CMEs during the data gaps of LASCO, or when the AR is near the central meridian or on the opposite side of the Sun, where Thomson scattering is less effective. To test such possibilities we have made a histogram of the selected CMEs in function of the longitude of AR 7978 relative to the central meridian (Fig. 7). Because of the relatively low number of CMEs, we have used bins with a 30 degree extension and we have summed up all the rotations. We also made a histogram of the data gaps of LASCO (Fig. 7) which allows us to correct the observed number of CMEs. Data gaps are defined by the absence of LASCO observations for three hours or more. For such period of time, a CME may have crossed the $\mathrm{C} 2$ field of view remaining unnoticed. During the entire period studied the data gaps amounted to 16 days. If we assume that the CME frequency is the same during observing times and data gaps we find that because of the absence of data we can only increase the number of CMEs associated to AR 7978 by a factor $\approx 10 \%$.

We now refine this estimate taking into account the time-dependence of the CME productivity. Both histograms of Fig. 7 show a clear peak around the West limb (longitudes 45-135 degrees) while a minimum is observed on the East limb (longitudes 225-315 degrees). The physics of the light scattering is the same in both cases so this difference should be an intrinsic property of AR 7978. The first flux emergence episode (in the first half of July) occurred dominantly when AR 7978 was on the western hemisphere and behind the West limb (see Sect. 2.2). Then the AR produced many CMEs, with a mean frequency of $0.6 \mathrm{CME} d a y^{-1}$. During the second flux emergence, which started while the AR was behind the limb but probably already on the eastern hemisphere, and lasted for about 10 days, we did not observe CMEs at all. However, there were regular data gaps during this period lasting 9 hours per day on average and adding up to 4.5 days. Supposing a similar CME frequency as during the first flux emergence period, we probably missed 23 CMEs around the East limb. During the decay phase of the AR the CME frequency decreased to $0.2 \mathrm{CME} \mathrm{day}^{-1}$. Since the total length of the data gaps during this period was 11.5 days, we may have missed 2 CMEs due to the gaps. All together, we find that $\approx 15-20 \%$ of all CMEs associated with AR 7978 are likely to have been missed.

While we clearly find this intrinsic temporal dependence in the CME production, the number of CMEs launched both towards (longitudes 315-360 and 045 degrees) and away (longitudes 135-225 degrees) from Earth is approximately the mean of the number of CMEs launched in the eastern and western quadrants! We conclude that we have no evidence that CMEs are underobserved for some phase angle. Additionally, CMEs from behind the solar limb, or CMEs which were not able to be definitely identified with the AR were not included. Therefore, considering both the data gaps and uncertainty in identification, the total number of CMEs identified may be underestimated by $1 / 3$.

\subsubsection{CME average helicity}

The largest uncertainty in the helicity ejected indeed comes from the average helicity that one CME can carry away. Presently, this estimate can only be done from the magnetic measurements in magnetic clouds. It is certainly an important hypothesis to suppose that the mean helicity contained in the CMEs of AR 7978 corresponds to the mean helicity contained in a set of 18 magnetic clouds observed at a different period of time. For example, if only the medium to large CMEs (15 observed associated to AR 7978, see Sect. 2.4) are large enough to correspond to the magnetic clouds, the helicity ejected by the CMEs would be reduced by a factor $\approx 0.6$. However, we have presently no quantitative means to relate the properties of the observed CMEs to those of the associated magnetic clouds. Then, consistent with DeVore (2000), we assume that the CMEs launched from AR 7978 carry in average a magnetic helicity corresponding to the 18 magnetic clouds analysed by Lepping et al. (1990). Additionally, none of the magnetic clouds observed by WIND/MFI correspond to the CMEs in our study, so explicit helicity comparisons are not possible.

Besides the intrinsic limitation of the model used to derive the global cloud parameters (see e.g. Vandas \& Geranios 2001, for a comparative study of a set of models fitted to the data of a magnetic cloud), the length of the magnetic cloud is an unknown. Above we have used the conventional value of 0.5 AU following DeVore's suggestion. But indeed, bidirectional flows observed in 

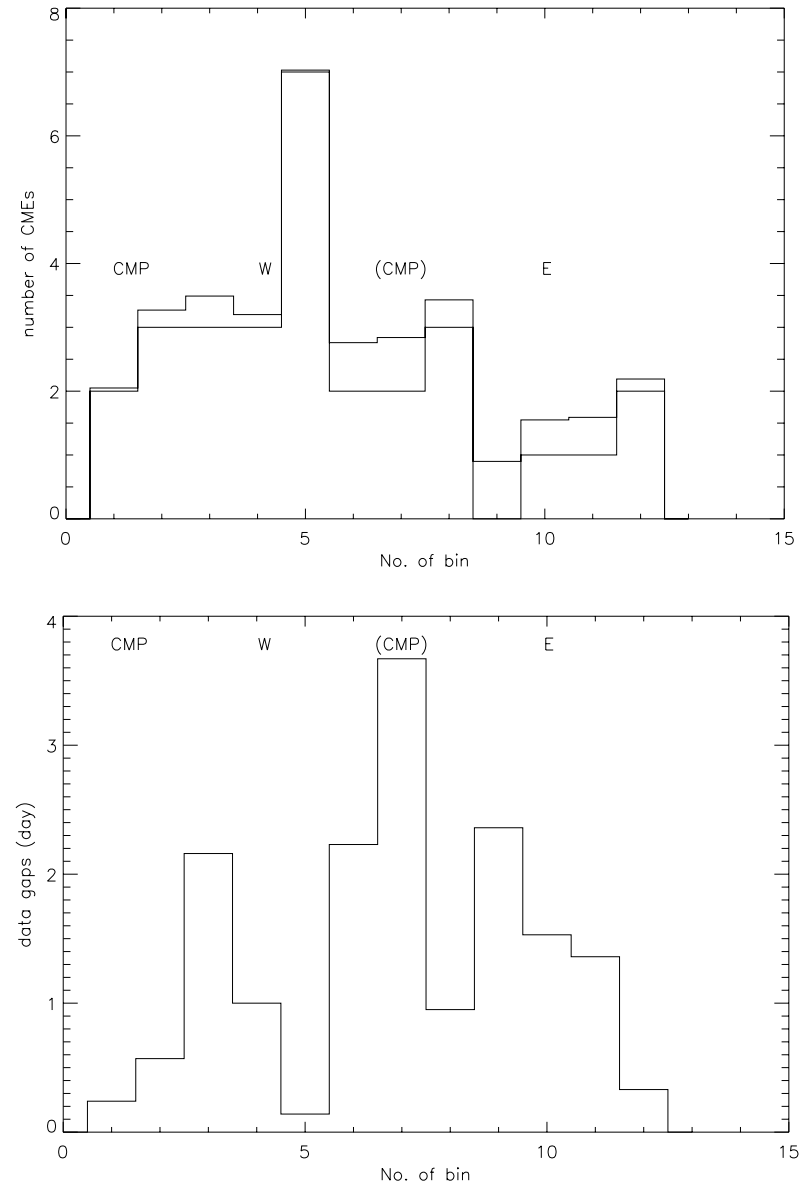

Fig. 7. Top figure. Thick line: histogram of the observed CMEs coming from AR 7978 summed in the period July-December 1996. In the abscissa the longitude of AR is plotted with 12 bins of 30 degrees (bin 1: \pm 15 degrees around CMP, bin 4: \pm 15 degrees around the West limb). The histogram with thin lines has been corrected because of the data gaps assuming that the frequency of the CMEs is the same during data gaps than during observing times (see Sect. 6.3). Bottom figure. Histogram for the fraction of time when no LASCO data were available (defined as periods with no data during more than 3 hours), so when CMEs could have been missed.

interplanetary clouds indicate that they are probably still rooted in the Sun when observed at 1 AU (e.g. Richardson et al. 1991; Richardson 1997; Shodhan et al. 2000). This implies that the length of clouds should be at least $2 \mathrm{AU}$, a factor 4 larger than DeVore's assumption. At this point it is worth remembering that the measurements of the magnetic field in clouds are local, and such local measurements are extrapolated to the full magnetic cloud. We may expect a uniform distribution of the twist along the cloud as a consequence of both magnetic flux and current conservation along the flux tube. Therefore, considering that magnetic clouds are still rooted in the Sun we can estimate a total ejected helicity $\approx 208 \times 10^{42} \mathrm{Mx}^{2}$, a factor $\approx 20$ larger than what differential rotation provides at most!

\section{Magnetic helicity budget in AR 7978}

\subsection{Helicity carried by emergence}

In Sects. 5.2, 6.2 and 6.3 we have shown that differential rotation cannot generate enough magnetic helicity to provide either the coronal helicity or the helicity ejected into the interplanetary medium. Another source of helicity is then required. From the AR evolution (Figs. 1-3) there are no obvious horizontal photospheric motions (like shearing ones) which can provide the needed helicity. We conclude that the helicity comes from the last term on the right hand side of Eq. (7). The budget of magnetic helicity per rotation writes:

$\Delta H_{\text {emergence }}=\Delta H_{\text {corona }}-\Delta H_{\text {diff.rot. }}+N . H_{\mathrm{CME}}$,

where $\Delta$ means the variation of the helicity, $N$ is the number of CMEs during the interval of time considered and $H_{\mathrm{CME}}$ is the mean helicity per CME. Unfortunately, in

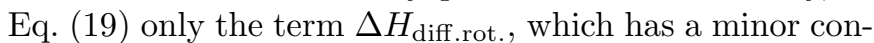
tribution, can be estimated with confidence; therefore, we can only discuss the general tendency below.

The variation of the coronal helicity, $\Delta H_{\text {corona, was }}$ first estimated from the minimum and maximum values derived with Eq. (13). Both values are coherent in magnitude, then we average them and subtract the contribution of the differential rotation. The last column of Table 4 gives this estimation of $\Delta H_{\text {corona }}-\Delta H_{\text {diff.rot. }}$ Then, the sum of the last two columns of Table 4 gives an estimate for $\Delta H_{\text {emergence. }}$ It indicates the need of a major injection of magnetic helicity during the first two rotations. This is coherent with the two episodes of flux emergence observed in July and August and tells us that most of the magnetic helicity indeed emerged from the convective zone.

The budget of helicity for the period September to November is less certain. Remembering the large errors that we may have on $\Delta H_{\text {corona }}$ and $N . H_{\mathrm{CME}}$, it is plausible that, during that period, the launch of CMEs simply depletes the coronal helicity without any need of injecting helicity from below $\left(\Delta H_{\text {emergence }} \approx 0\right)$. This would require that the helicity ejected on average in CMEs be low (lower than $2 \times 10^{42} \mathrm{Mx}^{2}$ ). If indeed magnetic clouds are more extended than $0.5 \mathrm{AU}$ at $1 \mathrm{AU}$ (because they are still linked to the Sun), their magnetic helicity could be much higher. In such a case the input of helicity from the convective zone should continue well after the "classical" emergence period (characterized by an increase of photospheric magnetic flux). There is no observational evidence in AR 7978 to support or reject this hypothesis.

\subsection{Physical mechanisms for long-term helicity injection}

The long-term input of magnetic helicity can be simply provided by the magnetic flux tube if it continues to move across the photosphere. This requires that it keeps some buoyancy and so that the magnetic field does not become strictly vertical in the vicinity and at the photosphere. 
We note that, when the upper section of the flux tube has fully crossed the photosphere, it is very difficult to infer the further emergence of a tube from observations (e.g. there is no appearance of new flux while no arch filament system and no detectable upward velocity are expected). It is only in the case when the emerging flux tube has a particular geometry (different from the classical $\Omega$ loop) that an indirect evidence of the long-term emergence can be obtained through the coherent evolution of the magnetic polarities. Such was the case in the long-term counter-kink rotation of one AR (López Fuentes et al. 2000) or in the U-loop geometry present in another AR (van Driel-Gesztelyi et al. 2000).

An alternative mechanism to this extended emergence, which does not require that the flux tube remains buoyant in the convective zone, has been considered by Longcope \& Welsch (2000). The twisted flux tube in the convective zone creates a net torque on the photospheric plasma which cannot be compensated by the plasma forces. After a transient period, during which torsional Alfvén waves are transferring twist to the corona, a steady state is reached, where the torque of the sub-photospheric flux tube is balanced by the torque of the coronal magnetic field. In short, for the steady state, the coronal helicity is determined by the amount of twist present in the subphotospheric part of the flux tube. Let us extend this model qualitatively to include CMEs. If the coronal helicity of the steady state is above the threshold of the global instability for the coronal field, a CME will occur, removing part of the helicity. Next, the imbalance of torque will again charge the coronal field with helicity typically in a day (see the time estimate of Longcope \& Welsch 2000) and, the process will start again. In this approach, the coronal field is permanently, but in a time dependent manner, filled by magnetic helicity from the convective zone until the flux tube twist is exhausted or the flux tube is destroyed by convective motions.

At present, observations do not let us decide whether the injection of magnetic helicity is limited or not to the "classical" emergence period. In order to probe this, a much more precise determination of the magnetic helicity is needed, in particular, of that contained in magnetic clouds. This could clearly constrain the mechanisms of flux emergence, but also the physics of CMEs, e.g. does the long-term persistence of the CME launch occur only because of the dispersion the AR photospheric magnetic field (leading to a coronal expansion of the magnetic configuration) or does it need a nearly continuous feeding from the convective zone?

\section{Conclusion}

The long-term evolution of a single active region on the Sun, with a large amount of data coverage during its six month evolution, was a unique opportunity to evaluate its magnetic helicity budget. The isolation of AR 7978, together with its simple bipolar nature, allows us to analyse an AR with a configuration closest to theoretical models. This clearly facilitates a quantitative analysis of the helicity budget (e.g. minimizing the exchange of helicity with a neighbor AR, and avoiding the possible caveat of multipolar configurations where several magnetic bipoles interact). We put emphasis on the magnetic helicity because, even in resistive MHD, it is dissipated on time scales much longer than six months. Magnetic helicity can then be used as a trace to understand the transport of the magnetic field from sub-photospheric layers to the corona, and then its ejection into the interplanetary medium.

The main question addressed in this study is whether magnetic helicity mainly is injected into the corona by horizontal photospheric motions or not. Considering the magnetic field evolution, the main candidate is the differential rotation. It provides a helicity injection which has the right sign for AR 7978 (compared to the inferred coronal helicity). We have estimated the magnetic helicity present in the coronal magnetic field of the AR. Even with the minimum values, the injection of helicity by differential rotation is too small, by at least a factor 2.5, to explain the coronal helicity (Sect. 5.2). Then, we have estimated the magnetic helicity carried away by CMEs assuming a one to one correspondence to magnetic clouds and using the mean values measured for the physical parameters of the clouds. We find that differential rotation provides a magnetic helicity too small by at least a factor of 4 (Sect. 6.2). This factor can be as high as 20 if the magnetic clouds are still attached to the Sun when their apex reach $1 \mathrm{AU}$ (Sect. 6.3). These two factors take into account only the CMEs observed with LASCO; their number might be slightly higher, up to $1 / 3$, due the data gaps and identification problems. We conclude that, contrary to DeVore (2000), differential rotation is not able to provide the magnetic helicity ejected from AR 7978, but that this helicity has to come dominantly from the convective zone.

We agree with DeVore (2000) on the amount of magnetic helicity injected by differential rotation and our conclusion, opposite to DeVore, comes only from the number of CMEs associated to AR 7978. This AR is indeed a typical AR, it has a magnetic flux close to the average flux value for an active region $\left(10^{22} \mathrm{Mx}\right.$, as used by Devore), but AR 7978 has a factor 5 more CMEs than the average value used by DeVore. This difference is mainly due to the much higher sensitivity of LASCO compared to previous instruments (e.g. aboard Skylab, P78-1 and Solar Maximum Mission), to the nearly continuous observations of LASCO (only $\approx 10 \%$ of data gaps) and also that at the minimum of the cycle different CME events can be more clearly distinguished.

The above estimates of the magnetic helicity show that the differential rotation can neither provide the required magnetic helicity to the coronal field (factor 2.5 to 4 ), nor to the field ejected to the interplanetary space (factor 4 to 20). It is instructive to rewrite the helicity in the "natural" units of $\Phi^{2}$, where $\Phi \approx 10^{22} \mathrm{Mx}$ is the magnetic flux of the active region. In this units, the maximum coronal helicity is in the interval $\approx[0.2,0.3]$, while the total helicity 
ejected is $\approx[0.5,2.0]$ and the total helicity input from differential rotation in AR 7978 is $\approx 0.1$ (which is nearly the maximum possible value). In the same units, the magnetic helicity of a twisted flux tube making $N$ turns is simply $N$ (see Eq. (A.5)). Then, the ejected magnetic helicity could originate from the twist of the flux tube which formed AR 7978.

Recent studies (e.g. Emonet \& Moreno-Insertis 1998) have shown that a minimum critical twist is needed so that a buoyant 2.5-D flux-tube is not destroyed during its rise by the hydrodynamic wakes that develop behind it. The pinch angle $\theta$ of the field should be typically larger than $\approx 20^{\circ}$ when it starts leaving the base of the convective zone. More recent studies do not significantly modify this result (see Abbett et al. 2000, and references therein). From this, one can estimate the length $L$ of the flux tube which has become unstable at the base of the convective zone and which later on has formed AR 7978, $L=2 \pi N a / \tan \theta$, where $N$ is the number of turns and $a$ is the flux tube radius. The radius $a$ can be simply estimated by using a typical field strength of $10^{5} \mathrm{G}$ for flux tubes becoming unstable at the tachocline (recalling that the magnetic flux $\Phi \approx 10^{22} \mathrm{Mx}$ ). We find for the unstable flux tube a length $L \approx 30 N \mathrm{Mm}$, showing that an $N$ of the order of a few units is indeed plausible with the mechanism of the ondulary (Parker) instability.

We conclude from the budget of magnetic helicity that the magnetic flux tube forming AR 7978 continues to emerge, or at least to provide an upward propagation of helicity to the corona (see the end of Sect. 7), for a much longer time than the observed photospheric increase of magnetic flux. One way to further constrain the duration of the emergence of flux tubes is to improve magnetic helicity estimations.

Acknowledgements. We thank C. R. DeVore and J. A. Klimchuk for useful discussions. P.D. and C.H.M. acknowledge financial support from ECOS (France) and SETCIP (Argentina) through their cooperative science program (A97U01). L.v.D.G. and Zs.K. were supported by the Hungarian Government grants OTKA T-026165, T-032846. P.D. L.v.D.G. and Zs.K. acknowledge the HungarianFrench S\&T cooperative program. The authors thank the SOHO/MDI, SOHO/EIT and SOHO/LASCO consortia for the SOHO data and the MSSL SURF for YOHKOH/SXT data. SOHO is a joint project by ESA and NASA. The CME catalog is generated and maintained by the Center for Solar Physics and Space Weather, The Catholic University of America in cooperation with the Naval Research Laboratory and NASA.

\section{Appendix A: Magnetic helicity of flux tubes in the convective zone}

Let's consider a section of a twisted flux tube and let us suppose, for simplicity, that the field has a local cylindrical symmetry together with axial invariance, then the magnetic field writes:

$$
\boldsymbol{B}=B_{\varphi}(r) \hat{u}_{\varphi}+B_{z}(r) \hat{u}_{z}
$$

where $r$ is the coordinate in the radial direction, $\hat{u}_{\varphi}$ and $\hat{u}_{z}$ are the unit vectors in the ortho-radial and axial direction. For simplicity, let us suppose that $B_{\varphi}=q r B_{z}$ where $q$ $(=\mathrm{d} \varphi / \mathrm{d} z)$ is uniform through the flux tube (this implies that field lines make the same number of turns per unit length along the axis independently from their distance $r$ to the axis). Then, the vector potential $\boldsymbol{A}$ is computed from Eq. (4) using the Coulomb gauge and $A_{\varphi}(r=0)=$ $A_{z}(r=0)=0$ :

$$
\begin{aligned}
& A_{\varphi}(r)=\frac{1}{2 \pi r} \Phi(r) \\
& A_{z}(r)=-\frac{q}{2 \pi} \Phi(r) .
\end{aligned}
$$

with $\Phi(r)=2 \pi \int_{0}^{r} B_{z} r \mathrm{~d} r$.

We can now compute the magnetic helicity in the volume $V$, which is a section of the cylinder $(0 \leq$ $r \leq R,-\Delta z / 2 \leq z \leq \Delta z / 2)$. Because the magnetic field crosses the boundary of the volume (at $z= \pm \Delta z / 2$ ), one should use Eq. (5) that defines the relative helicity. It is worth noting that using rather the initial definition of Eq. (3) (only suited for fields fully contained in a volume) gives the obviously wrong result of a null magnetic helicity (independently of the amount of twist)! However, Eq. (5) requires the reference helicity of the potential field. It is much easier to use a different, but equivalent, formulation for the relative helicity following Berger (1999). Let's write $\boldsymbol{B}$ as $\boldsymbol{B}_{\text {open }}+\boldsymbol{B}_{\text {closed }}$ where, in Berger's notation, "open" means a field crossing the boundary $S$ of $V$ and "closed" means a field confined inside the volume $V$. Berger (1999) shows that the relative magnetic helicity should be written as:

$H_{\mathrm{r}}=2 \int_{V} \boldsymbol{A}_{\text {open }} \cdot \boldsymbol{B}_{\text {closed }} \mathrm{d} V$.

It is worth emphasizing that an apparently similar integral: $2 \int_{V} \boldsymbol{A}_{\text {closed }}$. $\boldsymbol{B}_{\text {open }} \mathrm{d} V$ is not gauge invariant! For our purpose the "open" field is $B_{z} \hat{u}_{z}$ and the "closed" field is $B_{\varphi} \hat{u}_{\varphi}$. It follows directly from Eq. (A.4) that the relative helicity present per unit length in a twisted flux tube is:

$\frac{\mathrm{d} H_{r}}{\mathrm{~d} z}=\frac{\mathrm{d} \varphi}{\mathrm{d} z} \frac{\Phi^{2}(R)}{2 \pi}$

which is just the number of turns that field lines (at any radius) make per unit length multiplied by the square of the longitudinal magnetic flux. This is just the value expected intuitively, however this simple example shows how tricky helicity computations can be!

\section{References}

Abbett, W. P., Fisher, G. H., \& Fan, Y. 2000, ApJ, 540, 548 Alissandrakis, C. E. 1981, A\&A, 100, 197

Berger, M. A. 1984, Geophys. Astrophys. Fluid. Dyn., 30, 79 Berger, M. A. 1985, ApJS, 59, 433

Berger, M. A. 1986, Geophys. Astrophys. Fluid. Dyn., 34, 265

Berger, M. A. 1988, A\&A, 201, 355 
Berger, M. A. 1999, in Magnetic Helicity in Space and Laboratory Plasmas, Geophys. Monograph 111 (AGU, Washington DC), 1

Berger, M. A., \& Field, G. B. 1984, J. Fluid. Mech., 147, 133

Berger, M. A., \& Ruzmaikin, A. 2000, JGR, 105, 10481

Brueckner, G. E., Howard, R. A., Koomen, M. J., et al. 1995, Sol. Phys., 162, 357

Burlaga, L. F. 1988, JGR, 93, 7217

Burlaga, L. F., Sittler, E., Mariani, F., \& Schwenn, R. 1981, JGR, 86, 6673

Burlaga, L. F., Fitzenreiter, R., Lepping, R., et al. 1998, JGR, 103, 277

Chen, J. 1996, JGR, 101, 27499

Cliver, E. W., \& Hudson, H. S. 2001, JASTP, in press

Crooker, N., Joselyn, J. A., Feynman, J., (eds.) 1997, Coronal Mass Ejections, Geophys. Monograph 99 (AGU, Washington, DC)

Delaboudinière, J.-P., Artzner, G. E., Brunaud, J., et al. 1995, Sol. Phys., 162, 291

Démoulin, P., van Driel-Gesztelyi, L., Mandrini, C. H., López Fuentes, M., \& Aulanier, G. 2001, Sol. Phys., in press

DeVore, C. R. 2000, ApJ, 539, 944

Dryer, M., Andrews, M. D., Aurass, H., et al. 1998, Sol. Phys., 181,159

Emonet, T., \& Moreno-Insertis, F. 1998, ApJ, 492, 804

Finn, J. H., \& Antonsen, T. M., 1985, Comm. Plasma Phys. Contr. Fusion, 9, 111

Forbes, T. G. 2000, JGR, 105, A10, 23153

Forbes, T. G., \& Priest, E. R. 1995, ApJ, 446, 377

Gibson, S. E., \& Low, B. C. 1998, ApJ, 493, 460

González, W. D., Joselyn, J. A., Kamide, et al. 1994, JGR, 99,5771

González, W. D., Tsurutani, B. T., \& Clúa de González, A. L. 1999, Space Sci. Rev. 88, 529

Hidalgo, M. A., Cid, C., Medina, J., \& Vinas, A. F. 2000, Sol. Phys., 194, 165

Hudson, H. S., \& Cliver, E. W. 2001, JGR, in press

Hundhausen, A. 1999, The Many Faces of the Sun, ed. K. T. Strong, J. L. R. Saba, B. M. Haisch, \& J. T. Schmelz (Springer, New York, NY), 143

Klimchuk, J. A. 2000, Proc. of the Chapman Conference on Space Weather, AGU Monograph Series, ed. P. Song, G. Siscoe, \& H. Singer

Lepping, R. P., Burlaga, L. F., \& Jones, J. A. 1990, JGR, 95, 11957

Lepping, R. P., Burlaga, L. F., Ogilvie, K. W., Tsurutani, B. T., \& Lazarus, A. J. 1991, JGR, 96, 9425

Lepping, R. P., Burlaga, L. F., Szabo, A., et al. 1997, JGR, 102, 14049

Lin, J., Forbes, T. G., Isenberg, P. A., \& Démoulin, P. 1998, ApJ, 504, 1006

Longcope, D. W., \& Welsch, B. T. 2000, ApJ, 545, 1089

López Fuentes, M., Démoulin, P., Mandrini, C. H., \& van Driel-Gesztelyi, L. 2000, ApJ, 544, 540

Low, B. C. 1997, in Coronal mass ejections, Geophysical Monograph 99, ed. N. Crooker, J. A. Joselyn, \& J. Feynman (American Geophysical Press, Washington DC), 39

Mac Queen, R. M. 1980, Phil. Transac. of the Royal Soc. of London, A297, 605

Mac Queen, R. M., Csoeke-Poeckh, A., Hildner, E., et al. 1980, Sol. Phys., 65, 91
Marubashi, K. 1986, Adv. in Space Res. 6, 335 solar origins

Marubashi, K. 1997, in Coronal mass ejections, Geophysical Monograph 99, ed. N. Crooker, J. A. Joselyn, \& J. Feynman (American Geophysical Press, Washington DC), 147

Mandrini, C. H., van Driel-Gesztelyi, L., Thompson, B. J., et al. 2000, Geof. Inter., 39, 73

Mikić, Z., \& Linker, J. A. 1994, ApJ, 430, 898

Mikić, Z., \& Linker, J. A. 1997, in Coronal mass ejections, Geophysical Monograph 99, ed. N. Crooker, J. A. Joselyn, \& J. Feynman (American Geophysical Press, Washington DC), 57

Osherovich, V. A., \& Burlaga, L. F. 1997, in Coronal mass ejections, Geophysical Monograph 99, ed. N. Crooker, J. A. Joselyn, \& J. Feynman (American Geophysical Press, Washington DC), 157

Pevtsov, A. A., Canfield, R. C., \& Metcalf, T. R. 1995, ApJ, 440, L109

Pick, M., Démoulin, P., Maia, D., \& Plunkett, S. 1999, in Magnetic Fields and Solar Processes, European meeting on Solar Physics, ESA SP-448, 915

Richardson, I. G., Farrugia, C. J., \& Burlaga, L. F. 1991, in Lab. for High Energy Astroph. Contributions, 22nd International Cosmic Ray Conference, NASA, A92-36806 15-93, 9 field topology

Richardson, I. G. 1997, in Coronal mass ejections, Geophysical Monograph 99, ed. N. Crooker, J. A. Joselyn, \& J. Feynman (American Geophysical Press, Washington DC), 189

Scherrer, P. H., Bogart, R. S., Bush, R. I., et al. 1995, Sol. Phys., 162, 129

Schmieder, B., Démoulin, P., Aulanier, G., \& Golub, L. 1996, ApJ, 467, 881

Sheeley, Jr. N. R., Howard, R. A., Michels, T. J., \& Koomen, M. J. 1980, in Solar and Interplanetary Dynamics, ed. M. Dryer, \& E. Tandberg-Hanssen (D. Reidel, Hingham, MA), 55

Shimazu, H., \& Marubashi, K., 2000, JGR, 105, 2365

Shodhan, S., Crooker, N. U., Kahler, S. W., et al. 2000, JGR, 105,27261

Srivastava, N., González, W. D., González, A. L. C., \& Masuda, S. 1998, Sol. Phys., 183, 199 During 6-11 March 1993

Taylor, J. B. 1974, Phys. Rev. Lett., 33, 1139

Tsuneta, S., Acton, L., Bruner, M., et al. 1991, Sol. Phys., 136,37

Vandas, M., \& Geranios, A. 2001, JGR, 106, 1849

van Driel-Gesztelyi, L. 1998, in Three dimensional structure of solar active regions, Proc. 2nd. ASPE, ed. C. Alissandrakis, \& B. Schmieder, Astron. Soc. Pac. C. S., 155, 202

van Driel-Gesztelyi, L., Schmieder, B., Aulanier, G., et al. 1998, in New Perspectives on Solar Prominences, Proc. IAU Coll. 167, ed. D. F. Webb, B. Schmieder, \& D. M. Rust, Astron. Soc. Pac. C. S., 150, 366

van Driel-Gesztelyi, L., Thompson, B. J., Démoulin, P., et al. 1999b, in Plasma Dynamics and Diagnostics in the Solar Transition Region and Corona, Proc. SOHO-8 Workshop, ESA SP-446, 663

van Driel-Gesztelyi, L., Malherbe, J. M., \& Démoulin, P. 2000, A\&A, 364, 845

Webb, D. F. 2000, JASTP, 62, 1415

Webb, D. F., Cliver, E. W., Crooker, N. U., St. Cry, O. C., \& Thompson, B. J. 2000, JGR, 105, 7491 